\title{
Macht Wissenschaft glücklich? Wie Wissenschaft den Menschen kränkt, und wie sie dennoch zur Heiterkeit des Geistes beiträgt
}

\author{
Duddeck, Heinz
}

Veröffentlicht in:

Abhandlungen der Braunschweigischen Wissenschaftlichen Gesellschaft Band 51, 2001, S.93-109

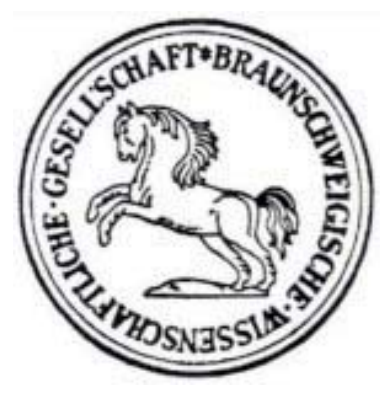

J. Cramer Verlag, Braunschweig 


\title{
Macht Wissenschaft glücklich? \\ Wie Wissenschaft den Menschen kränkt, und wie sie dennoch zur Heiterkeit des Geistes beiträgt
}

\author{
von Heinz Duddeck, Braunschweig*
}

Macht Wissenschaft glücklich? Eine törichte Frage? Denn Wissenschaft frage doch nur danach, wie etwas ist, was die Welt bewegt, sie im Innersten zusammenhält. Nicht nach der Befindlichkeit des Menschen, und schon gar nicht, wie er glücklich werde.

Etwas eingeschränkter gefragt: Macht das aus Wissenschaft gewonnene Wissen glücklich? Auch dies eine unzulässige Frage? Denn Glücklichsein ist ein Zustand der Seele, Wissen dagegen der Ertrag von Anstrengungen des Kopfes. Glücklich, das sind doch eher die Naiven, die Leichtköpfigen, die Verliebten etwa, - sagen die Schwerköpfigen, die da angekränkelt sind von der Gedanken Blässe. Macht wissenschaftliches Wissen eher unglücklich? Oder zumindest unfähiger, glücklich zu sein? Neiden etwa deshalb die Götter, die doch die Wissenderen sind, den Menschen das Glück?

Der Mensch kann offenbar glücklich sein, sonst strebte er nicht danach. Sind dagegen Götter glücklich? Ist Gott glücklich? Oder weniger gewagt gefragt: Ist Maria, dort wo sie heute ist, glücklich? Weil sie so wundersam helfen kann? Abermals törichte Fragen? Und warum sind Götter nicht glücklich? - Weil Glück das Leid als Pendant braucht?

Ich will davon reden, wie Wissenschaft das Selbstverständnis des Menschen kränkt und wie er sich dennoch die Heiterkeit des Geistes gewinnt. Die Heiterkeit des Geistes ist freilich noch nicht Glück, doch vielleicht die weiteste Annäherung daran, die über Wissen gewinnbar ist. Da begibt sich ein Ingenieur auf unsicheres Terrain. Dies wird kein wissenschaftlicher Vortrag. Eher eine Reflexion über die Konsequenzen wissenschaftlicher Ergebnisse. Fragen eher als Antworten. Lesefrüchte auch. Und was einem so durch den Kopf geht, wenn man - z. B. auch in Akademien - erfährt, was die Wissenschaften leisten und worauf sie zielen.

\section{Wissenschaft und Glück}

Wissenschaft lasse sich nicht mit Glück verbinden? Mitnichten. Wissenschaft, bei den Griechen die umfassender verstandene „Philosophie“, begann damit, zu fragen wie man glücklich werde. Aristoteles: „Glück ist das höchste Gut. Alles was der Mensch tut, ist darauf gerichtet. Lebensziel ist nicht Tugend um ihrer selbst willen, sondern ist Glück, Eudämonie, Glückseligkeit“. Dies steht bezeichnenderweise im 10. Buch der Nikomachischen Ethik. Moralisch handeln und glücklich sein, sei ein- und dasselbe. Glück

* Prof. Dr.-Ing.E.h., Dr.-Ing. Heinz Duddeck · Greifswaldstraße 38 - D-38124 Braunschweig Nach einem Vortrag am 12. Januar 2001. 
gehöre in die Kategorien der Ethik, nicht zur Psychologie. Das Erkennen der Weltordnung führe zum glücklichen Seelenzustand, denn die Seele ist Spiegel und Teil des Weltganzen. Philosophie ist bei den Griechen auch die Wissenschaft vom glücklichen Leben. Und schon vor Aristoteles meint Sokrates, also Platon: Es sei unmöglich, glücklich zu sein, wenn man gegen eigene Überzeugungen handle. Deshalb werde jemand, der weiß, was richtig ist, dies auch tun. Denn niemand möchte unglücklich sein. Also müsse man nur herausfinden, was für den Menschen gut ist, dann werde dieses Wissen zum Glück führen. Dies ist für uns heute reichlich naiv. Platon hatte noch nicht Freud gelesen. Der Mensch ist viel komplexer. Der Weg vom Wissen zum tugendhaften Tun ist weit, der vom Wissen zum Glücklichsein noch viel weiter.

Titus Lucretius (97 - 55 v. Chr.), von Epikur beeinflußt und von großer Wirkung auf die Renaissance, sagt in „De rerum natura“: Dem Glück und dem Seelenfrieden des Menschen stehen Götterfurcht, Aberglauben und Todesangst entgegen. Man müsse ihn durch rationales Wissen davon befreien. Die Seele sei sterblich. Es habe noch keinen Menschen gequält, tot zu sein. Wieso denn die Angst davor? - Also: Wissen befreit zum Glücklichsein?

Noch Kant definiert: „Das höchste in der Welt mögliche und . . . als Endzweck zu befördernde physische Gut ist die Glückseligkeit, unter der . . Einstimmung des Menschen mit dem Gesetze der Sittlichkeit, als der Würdigkeit, glücklich zu sein“ (Urteilskraft $\S 87)$. Das höchste Gut ist die Vereinigung von Tugend und Glückseligkeit.

Am Anfang der Wissenschaften, da fragten diese sehr wohl danach, wie der Mensch glücklich werde. Das Suchen nach Wissen hat die Glückseligkeit als Ziel. Aristoteles definiert drei Weisen des Glücklichseins in hierarchischer Ordnung:

1. Ein Leben der Lust und der Vergnügungen, was Sklaven und Tieren gemäß sei.

2. Ein Leben als freier, verantwortlicher Bürger in Tugend und Rechtschaffenheit, das auf Ehre zielt.

3. Die höchste Form des Glückes sei jedoch die als Forscher und Philosoph, die Selbstbetrachtung der Seele, die intellektuelle Suche nach Wahrheit durch den Gebrauch von Vernunft.

Es sei jedoch ein hinreichend Maß an Reichtum nötig, um Kontemplation zu ermöglichen. Der Gewinn von Wissen, also Wissenschaft, erzeuge Glückseligkeit. Diese Glückseligkeit ist nach innen gerichtet, nicht auf Sachen und Besitz. Sie ist ein Zustand des Geistes und der Seele.

Aristoteles fordert, daß der Staat, also die Politik, danach suchen solle, was dazu beiträgt, den Menschen glücklich zu machen. Dies steht dann auch in der Declaration of Independence der USA von 1776. Daß der Schöpfer den Menschen mit unveräußerlichen Rechten ausgestattet habe, wozu Leben, Freiheit und das Streben nach Glück gehören, und daß eine Regierung diese Rechte zu sichern habe. Die amerikanische Unabhängigkeitserklärung ist nicht nur ein Produkt der Aufklärung, des englischen Empirismus von John Locke und von Francis Hutcheson, der das sozialethische Prinzip des ,größtmöglichen Glücks für die größtmögliche Zahl“" einforderte. Die Unabhängigkeitserklärung greift auch auf griechisches Demokratie-Verständnis zurück: Regierungen sind nicht von Gottes Gna- 
den, wie Kaiser und Könige. Freilich, von allen politischen Idealen ist der Wunsch, die Menschen glücklich zu machen, vielleicht das gefährlichste, sagt Karl Popper.

Wo es Philosophen gibt, auch christliche wie Thomas von Aquin, gibt es auch das Suchen nach Glück. Die angebotenen Definitionen und Wege hierzu sind so vielseitig wie unsere 3000 Jahre alte Geistesgeschichte. Selbst der Begründer der neuzeitlichen Wissenschaften, Francis Bacon, sieht in der an das Gemeinwohl orientierten vita activa einen Weg zum Glück.

Leibniz schreibt, sittliches Handeln habe das Glück zum Ziel. Wenn sich der Einzelne vervollkommne, fördere er nicht nur sein eigenes Glück, sondern auch das Glück der Allgemeinheit. Hierin steckt die Idee des Fortschritts: Dem Menschen sei es aufgegeben, die Welt zum Besseren hin zu verändern. Die Aufklärung setzt dem christlich bedingten statischen Weltbild die Theorie des Fortschritts als Weg zu größerem Glück entgegen.

Der Plan der Schöpfung, wissenschaftlich nüchterner: die biologische Evolution, sieht jedoch nicht vor, den Menschen glücklich zu machen. Wahrscheinlich auch nicht den voll Lebenslust zwitschernden gefiederten Sänger auf der Tannenspitze. Das Glücklichsein des Menschen ist ein Produkt der Kultur. Also muß der Mensch sich seine Glückseligkeit erobern.

So, dies mag genug sein, um aufzuzeigen, daß die Humanwissenschaften sehr wohl darauf aus sind, Wege zum Glück zu finden. Da ist schon so viel vom Glück die Rede, daß Ludwig Marcuse, der andere Marcuse, auch spotten darf: „Ein glückliches Leben ist ein Leben, das frei ist vom hoffnungslosen Streben nach Glück“. Und Horaz (Epist. I.6.I sq) vermerkt: „Nichts zu bewundern, Numicius, ist wohl die einzige Sache, glückselig zu machen“. Diogenes klingt da an: Glück ist, nichts haben zu wollen.

\section{Parabel von wissenschaftlicher Welt und Lebenswelt}

„Der Arzt stellte fest, uns werden Zwillinge geboren“, sagt sie. „Komm, laß uns wie Faustus zu Demeter gehen“. ${ }^{1}$ Tief im Berg bei den Eleusischen Mysterien erscheint Demeter. „O, weise und große, allumfassende Göttin der Fruchtbarkeit gib unseren Kindern deinen Segen“. Da raunt es im heiligen Gewölbe: „Ihr kommet zu guter Zeit, denn heute jährt sich mein Geburtstag, an dem die Menschen mich erdachten, zum 3.000sten Male. So seien euch zwei Wünsche gewährt.“ Männer sind selbst vor Göttern vorlaut. Daher beginnt er: „Laß eines unserer Kinder alles Wissen der Welt begreifen, auf daß es weise wird“. - Sie sagt: „Laß mindestens eines von ihnen glücklich sein in und an der Welt, so wie sie ist".

Es gäbe auch einen Wunsch dazwischen: „Laß eines von ihnen so viel wissen, wie es braucht, die Welt mit eigenen Werken zum Besseren hin zu ändern. “Daraus wäre vielleicht ein Ingenieur geworden. Da es aber nun keine Drillinge waren... .

1 Sie weiß freilich nicht, daß Faustus nicht zu Demeter, sondern zu den "Müttern der Erde" in den sizilianischen Tempeln der Kreter ging. 
Und als 60 Jahre vergangen sind, sagt Felicitas zu ihrem Zwillingsbruder Sophos: „Komm, laß uns zu Demeter gehen, ihr zu danken“. Da raunt es wieder im heiligen Gewölbe der Erdgöttin: „So, ihr seid es. Nun, - habt ihre eure Gaben genutzet? O, ich sehe, Felicitas, du kannst mit dem Herzen denken, du hinterläßt eine goldene Spur. Warst du glücklich?“ „Eigentlich ja, Göttin, doch zuweilen nicht, wenn mein Herz zerbrach am Leid in dieser Welt.“ - „Und du, Sophos? O, ich sehe, du bist ein großer Wissenschaftler geworden, der die Natur- und die Lebenswissenschaften als Einheit versteht. Warst du glücklich?“ „Eigentlich nein, Göttin, doch zuweilen ja, wenn ich vergaß, wie die Wissenschaften den Menschen sehen.“- „Ja, ja“, sagt Demeter, „wo viel Wissen ist, da ist viel Grämens“.

Da mir die Parabel nicht so gut wie Lessing gelingt, muß ich kommentieren. Selbstverständlich weiß auch Felicitas wie Sophos,

- daß der so kurzlebige Mensch in die biologische Welt eingebettet ist,

- daß er ein Produkt der biologischen Evolution ist, die auch sein Denken umfaßt,

- daß sich das Weltall in 16 Milliarden Jahren entwickelt hat,

- daß die Sonne in 5 Milliarden Jahren ausgebrannt sein wird und

- daß sie dann die Erde in einem roten Feuerball vernichtet, mitsamt allen Kulturleistungen: Bach und Mozart, Dichter- und Philosophenworte, Wissenschaft und Technik und auch Mythen und Religionen.

Felicitas bringt sich dennoch, ihre 70 bis 80 Jahre Lebenszeit ganz bejahend, voll in ihre Lebenswelt ein. Sie ist dank Demeters Gaben auf natürlichste Weise - ohne den Umweg über ein Trotzdem - heiter, ja sogar glücklich und dabei ganz und gar nicht naiv. Wir jedoch hier im Saal? Waren etwa unsere Eltern bei Demeter? Also müssen wir uns schon selbst anstrengen, Heiterkeit und Glück zu finden.

Sophos hat es wegen der Wunschbitte seines vorlauten Vaters schwerer. Er weiß, was die Wissenschaft dem Selbstverständnis des Menschen angetan hat und noch immer antut. Daß z. B. alle Mythen von Weltwerdung und Lebenssinn wissenschaftlichem Fragen nicht standhalten. Sophos weiß, daß Weltall und Weltzeit den Menschen in Raum und Zeit so unendlich winzig machen. - Und er leidet daran. Obwohl doch Wissenschaft allein noch lange nicht das Ganze von Welterkenntnis ist. Er muß sich das Glück, die Heiterkeit in seiner Lebensspanne mit einem Trotzdem gewinnen. Daher Demeters: „Ja, ja, wo viel Wissen ist, da ist viel Grämens“. Bei Kohelet in Prediger Salomo 1,18 heißt es in Luthers Übersetzung: „Wo viel Weisheit ist, ist viel Grämens, und wer viel lernt, muß viel leiden." Hiermit ist nicht nur die Haltung des Intellektuellen gemeint, der naive Unwissenheit für eine Voraussetzung zum Glücklichsein hält. Ein glücklicher Intellektueller, dies sei ein Widerspruch in sich. Deutsche sind hierin übrigens Weltmeister. Nietzsche vermerkt: „Wer viel Freude hat, muß ein guter Mensch sein: Aber vielleicht ist er nicht der Klügste, obwohl er gerade das erreicht, was der Klügste mit all seiner Klugheit erstrebt". Nein, hier ist mit dem Trotzdem eher gemeint: die Sinnsuche in einer Welt, deren Sinn Wissenschaft nicht erschließt. Hans Blumenfelds Fragen gehören etwa dazu. Und auch diese Verszeilen: 
Zwei mal zwei gleich vier ist Wahrheit. Schade, daß sie leicht und leer ist, Denn ich wollte lieber Klarheit Über das, was voll und schwer ist.

Emsig sucht ich aufzufinden, Was im tiefsten Grunde wurzelt, Lief umher nach allen Winden Und bin oft dabei gepurzelt.

Endlich baut ich eine Hütte. Still nun zwischen ihren Wänden Sitz ich in der Welten Mitte, Unbekümmert um die Enden.

Wilhelm Busch ist der Autor. Und er meint mit „Enden“ das Woher, Wozu, Wohin des Menschen.

Und daß da in der Parabel Sophos der Wissenschaftler ist und Felicitas die LebensweltKluge, dies ist keine Geschlechter-Rollenverteilung. Einer von beiden mußte ja „mit dem Herzen denken können“. Dies können Frauen halt besser.

\section{Die Kränkungen des Selbstverständnisses des Menschen durch Wissenschaft}

Zum Glück des Menschen gehört auch das Verständnis seiner selbst: Was er in dieser Welt eigentlich sei, woher er komme und wohin er gehe. Daher sei nun aufgezeigt, wie Wissenschaft das Selbstverständnis des Menschen veränderte und noch fortwährend ändert. Ich will die wesentlichen Kränkungen des Menschen durch Ergebnisse wissenschaftlichen Forschens skizzieren, die schon Sigmund Freud ausmachte. Ob es tatsächlich Kränkungen für uns Heutige sind, mag der Leser für sich selbst abwägen. Historisch jedoch führten sie zu heftigsten Reaktionen.

\section{Kosmologie}

Die erste Kränkung ist uns allen wohl vertraut. Sie ist der Verlust des geozentrischen Weltbildes. Sie hat ihren Anfang in der kopernikanischen Erkenntnis, daß die Erde keineswegs Mittelpunkt und einzigartig und die ganze Welt ist. Helios steigt nicht am Morgen mit feurigen Rossen aus dem Okeanos. Der Sonnengott der Inkas braucht nicht das Opfer lebender Herzen, damit die Sonne der Erde erhalten bleibt.

Da läßt freilich schon Aristarchos von Milos (310 - 230 v. Chr.) die Erde um ein Feuer kreisen und erklärt Tag und Nacht mit der Rotation der Erde um ihre eigene Achse. Weil Aristoteles eine solche Hypothese jedoch verwirft, braucht es 1800 Jahre bis zu Kopernikus. Der kannte Aristarchos Schriften. Vor Kopernikus: Da zielt das ganze Welttheater der 
Natur, der Gestirne, der erlebbaren Geschichtlichkeit allein auf den Menschen. Nicht nur die Erde, sondern auch er ist Mittelpunkt der Welt. Und weil er so einzig und Zentrum ist, hat er auch eine privilegierte Stellung zu Gott. Da tut die kopernikanische Kränkung weh.

Kopernikus ahnt, wie die Kirche reagieren würde. Erst auf Drängen des 25jährigen Wittenbergers George Joachim Rheticus werden die sechs Bände „De revolutionibus orbium coelestium“ in Nürnberg gedruckt und gelangen 1543 wenige Stunden vor Kopernikus “ Tod in dessen Hände. Andreas Ossiander aus Leipzig fügt ein eigenes Vorwort hinzu, daß dies alles nur Hypothesen seien. Kopernikus widmet die Bände vorsorglich und demutsvoll Papst Paul III, der doch ein so großer Liebhaber der Wissenschaften sei. Die Katholische Kirche toleriert zunächst Kopernikus. Sein Werk kommt erst 70 Jahre später 1616 auf den Index und bleibt da bis 1835.

Martin Luther, Melanchthon und auch Calvin verurteilen Kopernikus scharf: Wer die Sonne anhält und die Erde in Bewegung setze, sei ein Narr, der die Schrift verhöhne. Giordano Bruno, der das Universum für unendlich erklärt mit vielen bewohnten Himmelskörpern, wird dafür - und weil er auch sonst die Kirche provozierte - im Jahre 1600 auf der Piazza dei Fiori in Rom öffentlich verbrannt. Und als Galilei 1633 sagt, daß das Kopernikanische Modell kein Modell sei, sondern die Wirklichkeit, da greift die Inquisition zu. Denn diese Kränkung von Bibelwort und menschlichem Selbstverständnis ist damals - für uns heute kaum nachvollziehbar - unerträglich groß, und ganz besonders für das Welt- und Menschenbild der christlichen Kirche. Wer jedoch die wissenschaftlichen Leistungen zur Welterkenntnis als Fortschritt bewundert, ist auch damals nicht gekränkt, sondern eher euphorisch.

Diese Kränkung hat sich in den 460 Jahren bis heute mit den Ergebnissen von Astronomie, Astrophysik und Kosmologie unserer Zeit sogar noch ungeheuer verschärft. Auch Kant wußte noch nicht, daß sein gestirnter Himmel über ihm aus nicht abzählbaren Spiralnebeln, aus Milliarden von Sonnensystemen besteht und etwa 16 Milliarden Jahre alt und weit ist. Das Hubble Space Teleskop kann bereits 10 Milliarden Jahre weit in die Vergangenheit hineinsehen. Die Astronomen sehen jedoch nur das, was schon Milliarden Jahre vergangen ist, niemals das Heute. Und wenn zukünftige Teleskope 16 Milliarden Jahre tief ins All reichen, müßten sie ja eigentlich den Urknall, den punktförmigen kleinen Anfang sehen. Da ist noch viel Widersprüchlichkeit zu erklären.

Was ist da der Mensch in einem solchen Weltall? Blaise Pascal sagt schon um 1650: „Das ewige Schweigen dieser unendlichen Räume macht mich schaudern.“ Und wir, nach 350 Jahren und mit so viel mehr an Wissen, was sollen wir erst schaudernd sagen? Die päpstlichen Verfolger von Galilei ahnten, welche Fragen die Kirche als Folge astronomischer Erkenntnisse zu beantworten hätte: Wie hat Gott dieses Staubkorn Erde in seinem in Zeit und Raum so unendlichen Weltall überhaupt gefunden und dann noch zur rechten Zeit des Erwachens menschlichen Geistes. Und aus dieser Unendlichkeit: eine so persönliche Offenbarung den Menschen des Alten Testamentes? Und wenn wir demnächst der Erde gleichende Planeten anderer Sterne entdecken: Haben Lebewesen dort auch Gottes Offenbarung erhalten? Der biblische Gott hat ja nicht einmal China gefunden.

Die Ergebnisse der Kosmologie reduzieren den Schöpfungsakt Gottes auf das Schaffen der physikalischen Grundgesetze in den ersten Millisekunden nach dem Urknall. Diese 
Naturkonstanten des physikalischen Universums (z.B. der Energieverhältnisse in Kernen der Kohlenstoffatome oder die Verhältnisse von Expansion zu Schwerkraft) sind so fein eingestellt, daß um wenige Prozente geänderte Konstanten weder Sterne noch Leben zuließen. Das Universum hat eine physikalische Struktur, die die Existenz von Lebewesen wohl zuläßt, jedoch nicht zwingend macht. So die schwache Fassung des Anthropischen Prinzips (R.K. Cliften und R.H. Dicke 1961). Die starke Fassung lautet: Das Universum ist so beschaffen, daß es unweigerlich Beobachter, also den Menschen, hervorbringt. Dies kann man so deuten, daß Leben und damit der Mensch nicht Zufall (wie bei der Formulierung der schwachen Fassung), sondern geplant sind. Einwand dagegen: Hätten die Dinosaurier Wissenschaft betreiben können, sie wären zu dem Schluß gekommen, die Natur habe auf Dinosaurier hingearbeitet. Für beide Fassungen des Prinzips würde gelten: Gott greife in den 16 Milliarden Jahren seit dem Urknall nicht in die Welt ein, er wache höchstens über das Bestehenbleiben der Gesetze. Gott mußte lange warten, bis er mit intelligenten Menschen wie Moses und Hiob reden konnte. Und mit der Naivität meines masurischen Großvaters gefragt: Wo eigentlich im unendlichen All wohnt Gott mit seinen Engeln und Heiligen, wo? Dies kann Wissenschaft nicht beantworten. - Vielleicht wohnt er in den Köpfen und Herzen der Menschen, - denn er ist ihnen so nahe.

Der Mensch erträgt diese kosmische Kränkung nicht. Er weicht ihr hundertfältig aus. Er ignoriert dieses Wissen in seiner Lebensumwelt. Er muß es vielleicht sogar, um Lebenszuversicht zu gewinnen. Wenn die Aussaat der Radieschen unter Maien-Vollmond eine reichere Ernte verspricht, dann wird auch heute noch die griechische Mondgöttin Selene um ihren Segen gebeten. Und der Ehepartner unserer Tochter soll doch bitte, bitte nicht ein Widder sein: Mythisches Erbe Babylons noch nach 2500 Jahren wirksam. Der Mensch bittet Götter um Wunder, also um das, was wissenschaftlichen Naturgesetzen widerspricht. Und siehe: es hilft. Wir leben offenbar gleichzeitig in allen Epochen unserer Kulturevolution. Da vertragen sich sogar Horoskope und Schwarze Löcher in Galaxien im gleichen Kopf. Wahrscheinlich ist es für unser Selbstgefühl gut, wenn wir verdrängen, wie einsam und klein der Mensch im Kosmos ist. Unserem normalen Lebensumfeld ist das so beängstigende Weltall schnuppe. Säßen wir hier etwa irgendwie anders beisammen, wenn die Welt geo - statt heliozentrisch wäre? - Doch Vorsicht: Dieses Wissen greift in unsere persönliche Sinngebung von Dasein und Leben ein.

\section{Evolution des Lebens}

Die zweite Kränkung ist die Entdeckung der Evolution des Lebens. Charles Darwins Selektionstheorie „On the origin of species by means of natural selection“ erscheint 1859 nach 20 jährigem Sammeln von Indizien. Sie modifiziert die vorangehenden Thesen von Jean de Lamarck (1741 - 1829). Darwin (1809 - 1882) wagt zunächst noch nicht, auch den Menschen einzubeziehen. Sein „The descent of man“ erscheint erst 1871. Dies tun davor schon Thomas Huxley (1825 - 1895) und Ernst Haeckel (1834 - 1919), er besonders vehement und gegen die biblische Genesis von Welt und Mensch. Darwin dagegen ist demütiger. Er sei Theist. Denn wenn sich der menschliche Geist aus so niedrigen Lebewe- 
sen entwickelt habe, wie könne man ihm da trauen, so weitreichende Schlüsse über die Schöpfung ziehen zu können.

Nach Darwin: Der Mensch nicht nur ein Nichts in Raum und Zeit des Alls, sondern nun auch noch: Nur eine der vielen Spezies, die in der biologischen Evolution aus erstem Leben vor 4 Milliarden und aus den ersten Einzellern vor zwei Milliarden Jahren zufällig entstanden sind. Diese Evolution ist blind. Sie hat keinen Plan eines Schöpfers. Leben ist nicht Teil des „Buches der Natur“ (Augustinus), in dem wir Gottes Geist lesen können. Der Schmetterling ist nicht ein Spiel der Schöpferlaune in Leichtheit und Schönheit. Gott hat den Menschen nicht vor 5761 Jahren (so der heutige jüdische Kalender), fertig und zum Herrschen über die Erde bestellt, in den Garten Eden gesetzt. - Er ist ganz und gar von dieser Welt.

Und mit den Ergebnissen der heutigen Evolutionsforschung: Alles, was wir sind: Körper, Verhaltensweise, aber auch das Gehirn, also das Denken, alles Zufallsprodukt allmählicher evolutionärer Anpassung!? Der ca. 150.000 Jahre alte Homo sapiens eine Fort- und Zweigentwicklung des Australopithecus afarensis vor 3 Millionen Jahren. Auch die Hominiden haben nur eine begrenzte Periode in der Evolutionsgeschichte? Wie die Dinosaurier? - Dies tut weh.

Das ist eine tiefe Kränkung. Noch heute, 130 Jahre nach Darwins „Descent of man“ und nach so vielfältigen wissenschaftlichen Beweisen entscheidet die Schulbehörde von Kansas (im August 1999), daß Darwin und der Big Bang nicht Lehrstoff sein dürfen. In sechs weiteren US-Staaten müssen die biblische Schöpfungsgeschichte und die Evolution gleichwertig gelehrt werden, denn Kindern sollte gesagt werden, daß sie nicht zufällig in der Welt seien. $44 \%$ der Amerikaner glauben an die Schöpfung, nur $10 \%$ halten die Evolution für wahr (Time-Magazin). Selbst Voltaire meinte, die Austern-Fossilien in den Pyrenäen könnten nur von der biblischen Sintflut stammen.

Vor Darwin: Da staunt der Homo sapiens noch über das Wunder seines Gehirns und hält sich - in allen Mythen und Religionen - für etwas ganz anderes als das Getier und Gewürm. Denn da er Gut und Böse unterscheiden, Vergangenheit und Zukunft denken könne, sei er wohl eher ein Ebenbild Gottes, das freilich der Erlösung und Gnade bedarf. Vor Darwin: Da fragt auch noch die Aufklärung, wie das Böse in die von Gott, so wie sie ist, geschaffene vollkommene Welt komme, z. B. Leibniz‘ Theodizee.

Nach Darwin: Da läßt sich das Böse als Überlebenskampf deuten, ,survival of the fittest“. Wenn der Mensch erkannt habe, daß sich Leben evolutionär entwickelte, dann könne er nun auch direkt in die Evolution eingreifen. Da ist der Schritt nicht weit zum Sozialdarwinismus, der mit Darwins natürlicher Selektionstheorie wenig zu tun hat. Er bleibt im angelsächsischen Raum noch theoretisch, z. B. mit Herbert Spencer (1820 1903), der eine gesellschaftliche Evolution durch Anpassung und Auslese schon vor Darwin vertritt. Im deutschen Sprachraum entstehen daraus Eugenik, Rassenhygiene, Rassenanthropologie. Hitler holt sich seine rassistischen Ideen aus sozialdarwinistischen Schriften. Oswald Spengler schreibt 1918 sein Buch: „Untergang des Abendlandes“.

Die biologische Evolution ist heute längst keine Hypothese mehr. Was Darwin noch nicht erklären konnte, die Entstehung völlig neuer Arten, die Sprünge in der Evolution, 
wird Mutation, Zufall und Selbstorganisation zugeschrieben. Die Evolutionstheorie sagt uns freilich nicht, wie Leben entstand, sondern nur, wie es sich entwickelte.

Die Genom-Forschung hat herausgefunden, daß alle zur Zeit lebenden Menschen, einschließlich der Aborigines in Australien zu 99,9\% die gleichen Gene haben. Sie stimmen zu 95 - $98 \%$ mit denen der Tierprimaten überein, zu $90 \%$ mit denen einer Maus und noch zu $60 \%$ mit den Genen einer Fliege. Sogar Bakterien sind unsere Gen-Cousinen. Über Abschätzungen der Mutationshäufigkeiten wissen wir über die Paläogenetik, daß die Hominiden sich vor etwa 5 Millionen Jahren von Primaten abzweigten. Die Evolutionswissenschaften haben freilich das Dilemma, daß sich Evolution nicht experimentell reproduzieren läßt. Gene mögen zwar körperliche Funktionen bestimmen, für Krankheiten verantwortlich sein. Sie sagen jedoch nichts über unsere Individualität aus. Hubert Markl: „Den Menschen mit seinem Gen-Satz gleichzusetzen, dies wäre eine Reduktion des Menschenwesens auf pure Polymer-Chemie“ (3/2000: „Dolly und die Folgen“). Die Medien irren, wenn sie die Gen-Forschung als Angriff auf die Würde des einzelnen darstellen. Gene entscheiden nicht darüber, was der Mensch ist und denkt. Wo kämen denn bei 99,9\% Gengleichheit die so verschiedenen Menschen her? Kulturevolution ist also genindifferent? Doch die Gen-Technik greift nun sehr gezielt in die biologische Evolution ein und fordert zu Entscheidungen in Ethikproblemen heraus, bei denen Wissenschaft wenig helfen kann.

Der Mensch setzt auf seine biologische Evolution die kulturelle. Die menschliche Evolution des Bewußtseins ist begleitet von einem Entwicklungsstrom, der zu einer kulturellen Evolution führt (Henri Bergson). Und die Folgen der Erkenntnisse der Wissenschaften von der kulturellen Evolution? Die Geschichtswissenschaften fragen auch nach Entstehung und Entwicklung von Mythen und religiösen Vorstellungen. Mircea Eliade zeigt auf, wie sich die Mythen prinzipiell gleichen. Der erwachende menschliche Geist sucht in allen Kulturen nach Erklärungen für Welt- und Menschwerdung und den Sinn von Leben. Mythen werden erzählt, um das Fragen nicht aufkommen zu lassen (Hans Poser). Wissenschaft fragt dennoch. Glaube lebt in Gewißheiten, fürchtet den Zweifel. Wissenschaft lebt vom Zweifel, sucht Gewißheiten. Da halten Mythen nicht stand. Bei Jan Assmann kann man in „Moses, der Ägypter“ nachlesen, daß die alttestamentarische Religion der Israeliten wahrscheinlich nichts anderes ist als eine anti-ägyptische Variante von Echnatons Monotheismus. Moses - so er nicht eine mythische Figur ist - sieht auf dem Sinai nicht Gott. Er bringt den Israeliten die großen Mysterien der Ägypter, reduziert auf eine Stammesreligion.

Haben auch Religionen ihre Evolutionsgeschichte? Buddha, Konfutse, Lao Tzu, die Autoren des Pentateuch, Zarathustra, Thales von Miletos sind nahezu Zeitgenossen. Karl Jaspers nennt es die Achsenzeit. Als ob der menschliche Geist in allen Weltteilen zugleich die gleiche Entwicklungsstufe erreicht. Wenn die Wissenschaft in Jehova den Vorgänger Baal entdeckt, in Christus einiges vom essenischen Messias, dann entzieht sie Religionen ihre Einzigartigkeit in Zeit und Welt. Werden nicht auch wir Laien skeptisch, wenn wir auf Reisen anderem Glauben begegnen? Da relativieren sich lokale Glaubenssachen. Auch religiöse Weltbilder sind offenbar evolutionäre Kulturschöpfungen des Menschen. Und 
damit ethische Wertvorstellungen, wie Mensch und Welt sein sollen, auch? Vielleicht entwickelt sich in Zukunft gar Wissenschaft als neuer Glaubensmythos (Dieter Simon).

Der Mensch weicht dieser zweiten Kränkung, ein Zufallsprodukt der Evolution zu sein und die Mythen von Welt- und Menschwerdung zu verlieren, in seiner Lebenswelt auf vielfältige Weise aus. Und dies fällt ihm leicht. Denn wenn unser Kopf nur das denken kann, was und wie er in der Evolution zu denken gelernt hat, wie soll er da gekränkt sein, daß er so - vielleicht allzu einfach gestrickt - denkt. Wir leben - auch intellektuell - in einer von uns geschaffenen Kulturwelt, die die Evolution ignoriert. Wir bestaunen die Schönheit eines Gebirgstals als Gottesnatur, obwohl wir wissen, daß Gletscher es gefräst haben. Christen sind trotz des Evolutionswissens in einer tiefen Geborgenheit, denn sie wissen, woher sie kommen, was sie sollen, wohin sie gehen. Wir fragen nicht, ob Gott auch schon die Seele eines archaischen Homo sapiens zu sich aufnahm.

Die kulturelle Evolution hat die biologische Evolution für den Menschen sogar außer Kraft gesetzt. Suchen wir etwa unseren Lebenspartner für das Zeugen von Kindern nach darwinschen Selektionskriterien aus? Nicht einmal die Frauen, die ja zu dem Geschlecht der darwin-aktiveren Weibchen gehören. Doch die Evolution steckt irgendwie in uns drin. Denn wir lieben unsere biologischen Verwandten, den Teddybär und Frederik die Maus, nicht aber Kröten und Spinnen.

\section{Psyche und Seele}

Die dritte Kränkung beginnt mit Schopenhauers Urwillen und Freuds Aufdecken des psychisch Unbewußten. Der Mensch sei gar nicht Herr im eigenen Hause. Schopenhauer: Handlungsfreiheit ist nicht Willensfreiheit, denn wir können tun was wir wollen, aber nicht frei wollen, was wir unbewußt wollen. Sigmund Freud findet ein Es in uns, den dunklen, unzugänglichen Teil unserer Persönlichkeit, das Zentrum unseres aus der Evolution ererbten Lusttriebes. Dieses Es kennt keine Werte, nicht Gut noch Böse, keine Moral, sagt Thomas Mann. Das Ich liege mit dem Es im Dauerkonflikt, da das Ich dem Es ständig ein Zensor zu Kultur hin sein müsse. Das Gewissen des Über-Ichs zeige den Weg dazu.

Der Mensch nicht nur einsam im All, eine zufällige Species auf Erden, sondern nun auch noch ohne Willens- und Handlungsfreiheit, ohne das, worauf er seit Evas Apfel im Grunde so stolz war. Wie kann er da glückselig sein, wenn er sich so sehr psychoanalytisch argwöhnisch über die Schulter schaut.

Sigmund Freud hat die Wissenschaften weniger, dafür um so mehr Künstler und Schriftsteller beeinflußt. Doch sein Thema, Bewußtsein und Willensfreiheit, ist heute Ziel sogar der Naturwissenschaften, der Neurowissenschaften geworden. Sie wollen herausfinden, was in den Neuronen und ihren Kontaktstellen, den Synapsen, im Gehirn vorgeht, wenn der Mensch denkt, handelt, spricht oder fühlt. „Ein Hirnforscher versucht zu verstehen, was er da versteht, wenn er versteht", sagt Dieter Simon. Bevor Wolf Singer uns in der Berliner Akademie erklärt, wie sich jedem Denk- und Handlungsprozeß neuronale Prozes- 
se zuordnen lassen. Experimentell lasse sich nachweisen, daß Handlungsfreiheit eine Illusion sein kann. „Wir tun nicht, was wir wollen, sondern wir wollen, was wir tun“, sagt Wolfgang Prinz. Auch dazu gibt es etwas bei Wilhelm Busch:

\section{Der Knoten}

Als ich in Jugendtagen

Noch ohne Grübelei,

Da meint ich mit Behagen,

Mein Denken wäre frei.

Seitdem hab ich die Stirne

Oft auf die Hand gestützt

Und fand, daß im Gehirne

Ein harter Knoten sitzt.

Mein Stolz der wurde kleiner.

Ich merkte mit Verdruß:

Es kann doch unsereiner

Nur denken, wie er muß.

Nun gut, sagen die Geisteswissenschaften, die Neurowissenschaft mag wohl finden wie gedacht, aber noch lange nicht, was gedacht wird. Und erst recht noch nicht, wie das mysteriöse und doch so reale Ich-Bewußtsein in den Kopf kommt und in die Seele, himmelhoch jauchzend, zu Tode betrübt. Die Vorstellung eines rein neuronalen Gehirns, das „Entscheidungen trifft“, sei genauso absurd wie die eines Konzertflügels, der eine Beethoven-Sonate komponiert (U. Runge u. W. Wagner, Lehre u. Forschung 8/2000). Da wird noch lange gestritten werden, was Geist und Seele seien und wie sie in uns wirken. Wenn das Gehirn des Menschen so einfach wäre, daß er es verstehen könnte, dann wären wir so dumm, daß wir es doch nicht verstehen würden (Jostein Gaarder in „Sofies Welt“, S. 392).

Die Neuro- und Kognitionsforschung ist dabei aufzuzeigen, daß Denkvermögen, Bewußtsein, seelische Empfindungen neuronal erklärbar sein werden. Damit stellt Wissenschaft nicht nur Willensfreiheit, sondern auch Geist und Seele in Frage. „Das Ich ist nicht der „Herr im Hause“, sondern ein Konstrukt des Gehirns zur besseren Planung und Ausführung komplexer Handlungen“, so Gerhard Roth, Bremen (Forschung u. Lehre 5/2000). Die Neurobiologie macht sich auf, sogar die Seele als neuro-physiko-biochemischen Prozeß zu erklären; daß Leib und Seele gar nicht trennbar seien und auch der Geist kein epikuräisches Pneuma. Doch vielleicht werden durch diese Forschungen nur die prinzipiellen Grenzen einer rein naturwissenschaftlichen Erklärung von Bewußtsein sichtbar.

Da bahnt sich eine weitere tiefe Kränkung an. Denn der Mensch kann doch über sich hinaus denken, Vollkommenes sehen, Gut und Böse unterscheiden, Freude und Leid (auch fremdes) empfinden. Also müsse - so schon Homer und die Pythagoreer - der Mensch eine unvergängliche Seele im sterblichen Körper haben. Ägypter und Israeliten dagegen 
trennen noch nicht Leib und Seele. Pythagoras (ca. 570 - 480 v. Chr.) hält die Seele für das wahre unsterbliche Wesen des Menschen. Der Leib sei nur ihre vorübergehende vergängliche Behausung. Die Seele jedoch sei göttlicher Abstammung und bewege sich auf einem Rad der Wiedergeburt, um an ihren Ursprung zurückzukehren. Die griechische Seele ist über Paulus in das Christentum eingegangen. Sie bekommt von Augustinus zusätzlich einen diesseitigen Bewährungsauftrag, um über Strafe oder Lohn im Jenseits zu entscheiden.

Auch diese dritte Kränkung nehmen wir nicht an. So leicht geben wir unsere liebgewordene Denkgewohnheit nicht auf, daß wir da etwas in uns haben, das so anders ist als der biologische Körper, nämlich freier Wille, Bewußtsein, seelische Reaktionen, Geist. Freilich, wieso verflüchtigt sich Geist so rasch, wenn bohrender Zahnschmerz einsetzt?

Wir dürfen die Ergebnisse der Neurowissenschaften in unserer Lebenswelt getrost ignorieren. Denn selbst wenn Bewußtsein und Geist sich tatsächlich irgendwann mit einer Theorie von Informationsverarbeitung im Gehirn erklären lassen. Dies ändert nichts an unserem subjektiven Erleben von Glück und Trauer, Schönheit und Liebe. Geist und Seele werden weiterhin unsere sprachlichen Metaphern bleiben, nicht nur in Dichterworten wie hier bei Rilke:

Schau ich die blaue Nacht, vom Mai verschneit, in der die Welten weite Wege reisen, mir ist: Ich trage ein Stück Ewigkeit in meiner Brust. Das rüttelt und das schreit und will hinauf und will mit ihnen kreisen ... Und das ist Seele.

Und wenn Wissenschaft die unsterbliche Seele nicht findet? Wenn sich die Erfinder der Seele irrten? - Wissenschaft sei doch halt nur eine Teilmenge aller Wege zu Erkenntnis und Wissen, sagt da unsere gekränkte Seele.

So, dies ist fast schon zu viel über die Kränkungen des Selbstverständnisses des Menschen. Uns fällt es heute leicht, sich nicht durch Wissenschaft kränken zu lassen. Wir leben nicht mehr in der Hybris, der Mensch sei Mitte der Welt und zugleich nicht von dieser Welt. Vielleicht war dies nur der Traum von einem ganz anderen Menschen zu der Zeit, als des Menschen Phantasie erwachte. Ein Traum, auch den des verheißenen Glücks im Jenseits. Vielleicht ist es auch anders: Was Wissenschaft über Weltall, Evolution und Gehirn herausfindet, ist unserer Lebenswelt so fern, da können wir unbeschadet so leben, denken und handeln, wie der alte Adam sich die Welt zurecht legte.

\section{Heiterkeit des Geistes}

Vor lauter Kränkungen haben wir das Glück aus den Augen verloren. Nun muß ich ja endlich zur Heiterkeit des Geistes kommen. Ich zitiere: „Wer heiter ist, hat immer Ursache es zu sein, nämlich die, daß er heiter ist. Ist einer reich, jung, schön, geehrt: so frägt sich's, ob er dabei heiter ist ... Umgekehrt aber ist er heiter, so ist's einerlei, ob er jung oder alt, arm oder reich ist: Er ist glücklich. Was uns am unmittelbarsten beglückt, ist die Heiterkeit 
des Sinnes, denn diese gute Eigenschaft belohnt sich augenblicklich selbst“. So, Schopenhauer in seiner Eudaimonologie „Die Kunst, glücklich zu sein“. Der, der doch sonst Glück nur als Abwesenheit von Leid definiert. Philosophie fragt auch mit ihren pessimistischen Vertretern, wie der Mensch glücklich werde. Es müsse aber noch etwas hinzukommen, sagt Schopenhauer. Heiterkeit benötige äußere Glücksumstände und vor allem einen ,,hohen Grad vollkommener Gesundheit“. Heiterkeit erfordere „Vermeidung aller Ausschweifungen, auch aller heftigen oder unangenehmen Gemütsbewegungen, auch aller großen und fortgesetzten Geistesanstrengungen; endlich täglich wenigstens zwei Stunden rascher Bewegung in freier Luft". (Lebensregel Nr. 13).

Diese Schopenhauersche Heiterkeit ist unmittelbare, vom Charakter geschenkte Heiterkeit, die zusätzlich ein entsprechendes Verhalten erfordert. Felicitas ' Heiterkeit, von Demeter geschenkt, ist eher eine solche. Ich meine, es gibt noch eine andere, die aus unserer Kultur erwächst, eine Heiterkeit, die nicht geschenkt wird, wohl aber gewinnbar ist, vielleicht auch erst mit einem Dennoch gegen die Welt, so wie sie ist.

Macht Wissenschaft glücklich? Da könnte man zunächst an den Wissenschaftler selbst denken. Selbstverständlich macht das Finden einer erklärenden Theorie, das Erkennen von einfacheren Zusammenhängen in der Vielfalt der Erscheinungen, glücklich. Wenn Watson und Crick die Doppelhelix für die Struktur des DNS-Nucleinsäure-Moleküls in den Chromosomen finden. Wenn James Maxwell die elektro-magnetischen Erscheinungen in seinen Feldgleichungen mathematisch beschreiben kann. Wenn Einstein den Ausweg aus Widersprüchen gemessener Lichtgeschwindigkeiten durch seine Relativitätstheorie findet. Doch dies ist das Glücksgefühl des Wissensgewinns. Als Goethe den Zwischenkieferknochen entdeckt, schreibt er an Frau von Stein: „Ich habe eine solche Freude, daß sich mir alle Eingeweide bewegen“. Und Herder erhält jubelnde Briefe. Als sich jedoch herausstellt, daß ein französischer Anatom Vicq-d'Azyr das „Knöchelchen“ schon acht Jahre vorher entdeckt hatte, gibt es eifersüchtige Prioriäten-Streitereien. Auf seine Farbenlehre ist er so überzogen stolz, daß er Newton mit seinen Prismen verunglimpfend zum persönlichen Feind hochstilisiert. Selbst-Finden macht glücklich; was andere finden nicht. Dieses Glück des Erfolgs unterscheidet sich jedoch kaum von dem Leistungsstolz eines Tischlers, dem ein Möbelstück gut gelingt, eines Ingenieurs über sein Werk oder - würde Neil Postman sagen - von dem Erfolgsglück, das Tante Molly erfährt, wenn der Kuchen nach dem neuen Rezept prächtig gelingt.

\section{Definition von Heiterkeit}

Machen die Ergebnisse von Wissenschaft, macht Wissen heiter? So direkt sicherlich nicht, eher indirekt. Ich will auf die Heiterkeit des Geistes hinaus. Also muß ich versuchen, sie zu definieren. Da helfen Aufsätze von Wilhelm Schmid (ZEIT 7.11.99) und Michael Erler (ZEIT 1.7.99) und die Philosophen der Klassik: Demokrit, Epikur, der Stoiker Seneca.

Wenn ich eine Zusammenfassung wage: Heiterkeit ist die gelassene Wohlgestimmtheit der Seele, zu der der Kopf angstbefreiendes Wissen und Disziplin, zu der Wissenschaft und Technik ein ausreichendes Maß an Gesundheit und Wohlergehen beitragen. 
Welches Wissen ist nötig?

1. Für weitgehend alles gibt es natürliche Erklärungen. Weder übelgelaunte noch wohlwollende Götter greifen in diese Welt mit Strafen oder Wundern ein. Es gibt keine Strafandrohungen oder Heilsversprechungen aus der anderen Welt. Leben ist nicht Bewährungsprobe für jenseitige Glückseligkeit. Fürchte die Götter nicht, sagt Epikur.

2. Die Dreizehn ist eine ganz normale Primzahl. Schwarze Katzen von links bestimmen nicht den Tagesablauf. In den Sternen steht kein Menschenschicksal.

3. Der Tod ist wie die Geburt das Natürlichste der Welt. Die Angst vor dem Tod ist schlimmer als der Tod, sagt Epikur.

4. Krankheit, Schmerz und Leid gehören zum Leben wie das Atmen. Doch Wissenschaft kann sie erträglicher machen.

5. Gegen Gefährdungen der eigenen Existenz helfen weder Technik noch Psychologie. Das Abgründige ist des Glückes Nachbar. Auch die Gefährdung unserer Welt durch z. B. die ungehemmte Beschleunigung von Technik ist eher Aufgabe zur Änderung als apokalyptisches Schicksal.

Dies das nötige Wissen, wozu Wissenschaft viel beträgt. Und welches Verhalten verhilft zur Heiterkeit?

1. Sei maßvoll in Wünschen und Lüsten. Lebe symmetrisch zwischen dem Zuviel und Zuwenig, sagt Demokrit.

2. Nimm Dinge und Menschen so, wie sie sind.

3. Lerne dich tatsächlich zu freuen über Erfreuliches, sagt Epikur.

4. Üb dich in heiter ironischer Distanzierung zu allem, was dich ärgern könnte. Halte Distanz zu der Welt um dich. Thomas Mann schreibt in dieser ironischen Heiterkeit der Distanz. Vom Bergesgipfel der Heiterkeit wird all das da unten im Nebel zum „Gehudel und Gesudel der Welt“.

Wenn man all dies weiß und kann, komme man der gelassenen Wohlgestimmtheit der Seele näher. Die heitere Seele besitzt sich selbst, auch unangefochten ,mitten im Sturm“. Sie ist auf das Negative vorbereitet, dennoch frei von ängstlicher Sorge, ja sogar frei von Angst vor der Angst. Sie ist resistent gegen Enttäuschungen. Die Heiterkeit ist ,skeptisch gegen die Gewißheit, ohne unter der Ungewißheit übermäßig zu leiden“, so Wilhelm Schmid.

Heiterkeit ist nicht Fröhlichkeit, auch nicht die ernstere des „O du fröhliche“ zu Weihnachten. Fröhlichkeit drückt Freude über ein Ereignis oder ausgelassene Unbeschwertheit aus. Heiterkeit ist mehr als Zufriedenheit, die auf Befriedigung von Wünschen und Bedürfnissen zielt. Ein muttermilchvoller Säugling fällt in den Schlaf satter Zufriedenheit. Er weiß noch nichts von der Heiterkeit des Geistes. Heiterkeit ist nicht Glückseligkeit. Diese jauchzt vor Glück. Heiterkeit lächelt nach innen. Wie in der Kunst es ferne Götter so unübertroffen tun: Ramses II am Eingang zum Luxor-Tempel; ein gelungenes Buddha-Antlitz, das die Heiterkeit tiefen Friedens und innerer Freiheit im Glückszustand des Nirwana einfängt. Das Bild des Gekreuzigten kann und darf dies nicht, insbesondere nicht nach der Gotik, Grünewalds Isenheimer Altar. Doch Heiterkeit kann sehr wohl aus Glaubensgewißheit wachsen. 
Wenn man soviel sagen muß, um Heiterkeit zu definieren, ist dies offenbar nicht leicht. Und ich weiß wohl, daß ich hier Epikur recht nahe bin. Doch darf ich's etwa nicht? Epikur überschätzt wahrscheinlich, was der Verstand vermag. Irgendwo fand ich: „Was könnte wichtiger sein als das Wissen, fragt der Verstand. Das Gefühl will mit dem Herzen sehen, antwortet die Seele“.

\section{Was Wissenschaft dazu beiträgt}

Und was trägt die Wissenschaft zur Heiterkeit bei? Ich redete gar lange über die Kränkungen durch die Wissenschaften, weil ich zugleich auch aufzeigen wollte, wohin Wissenschaft auf diesen drei Feldern zielt. Dabei sollte eigentlich sichtbar geworden sein, daß die Ergebnisse von Wissenschaft den Menschen nur da kränken, wo er noch mythische Vorstellungen von sich und der Welt hat. Er weiß zu wenig, daher ist er gekränkt. Wissenschaft will mehr wissen und schert sich nicht um Kränkungen der Unwissenden. Diogenes redet von der „Pest der Unwissenheit““.

Wissenschaft und Technik wollen befreien. Am Anfang noch von den Fesseln der Natur wie Hunger, Kälte, Krankheit, Mühsal. Und diese Befreiung ist ja zweifelsfrei sehr erfolgreich gelungen. Wissenschaft kann Leben sichern, Schmerz lindern, macht Technik möglich, bringt Ordnung in die Vielfalt. Wissenschaft befreit von Ängsten: Blitz und Donner sind nicht Götter-Zorn. Es gibt keine Dämonen und Gespenster. Ein Hexenglaube ist eigentlich nicht mehr möglich.

Heute will Wissenschaft auch befreien zu einem Mehr an Kultur. Nietzsche spottet: „Die moderne Wissenschaft hat als Ziel: So wenig Schmerz wie möglich, so lange leben wie möglich, - also eine Art von ewiger Seligkeit, freilich eine sehr bescheidene im Vergleich mit den Verheißungen der Religionen“. Was staunte wohl Nietzsche, wenn er sähe, was Wissenschaft seit 1889 zur Befreiung des Menschen leistete. Allein die Angst vorm Zahnziehen damals. Er sagt aber auch - und meint die Heiterkeit des Geistes: „Eins muß man haben: entweder einen von Natur aus leichten Sinn oder einen durch Kunst und Wissen erleichterten Sinn." Wissen also sei nötig und die Kunst.

Der Homo sapiens hat sich mit den Fähigkeiten zu Sprache, Denken, Phantasie und Geist - und mit der Fähigkeit zu Wissenschaften - in dieser so gar nicht auf des Menschen Glück bedachten Natur eine Kultur geschaffen, die die Heiterkeit ermöglicht. Wir können wegen der befreienden Leistungen dieser Kultur - wenn auch nicht gleich glücklich sein so doch die Heiterkeit des Geistes gewinnen. Aus Wort und Schrift, Wissenschaft und Technik, aus Kunst und Musik und den vielen anderen Kulturleistungen entspringt eine Freiheit von Geist und Seele, der Gipfelblick über Berg und Tal, die heiter machen. Unser Geist hat offenbar die Fähigkeit, das Gehäuse, worin er steckt, die körperliche Existenz, vergessen zu machen, sich leicht und frei und unbeschwert seiner selbst zu erfreuen. Diese Heiterkeit des Geistes meine ich. Heiterkeit, das ist das vergeistigte Glück, das nicht aus der Naivität kommt. Und wenn ich mir da Bestätigung hole, bei Heinrich Heine: „Die Herrlichkeit der Welt ist immer adäquat der Herrlichkeit des Geistes, der sie betrachtet.“ 


\section{Schluß-Parabel}

In 100 Jahren von heute, da lesen zwei Singles meine Eingangsparabel im Internet. Und sie machen sich auf nach Sizilien, dort wo Faustus tatsächlich war. "Laß uns zu den Müttern der Erde gehen. Vielleicht ist auch Demeter da. Ihr unser Leid zu klagen”. Nach vielem Fragen und Suchen finden sie endlich unter den 25 restlichen Säulen des HeraTempels in Agrigento den Zugang zu den Grotten der einst kretischen Tempel.

„Hello, Demeter, do you hear us? Bist du hier? Gibt es dich noch?“. Saloppe Töne in heiligen Gewölben. - Da dauert es. - Bis endlich eine Antwort grummelt: „Was wollt Ihr? Habt ihr keinen Respekt vor Gottheiten, selbst wenn sie Mythen sind?“ - Dies hilft. - „O, große Göttin, Tochter des Kronos, Mutter der Persephone, wir bitten dich um Rat. Die Wissenschaften erklärten uns die ganze Welt. Sie befreiten uns von Dämonen und Gottesfurcht. Krankheit und Tod ängstigen uns nicht mehr. Doch irgend etwas fehlt. Sag, o Göttin, wie werden wir glücklich - oder zumindest heiter?““

Nach so vielen Jahren des Schweigens wird Demeter gesprächig: ,Vielleicht wißt ihr zuviel?“ - „Nein“, sagt er, „Wir haben wohl längst herausgefunden, wie unser Kopf denkt, wenn er denkt. Doch wir wissen nicht, woher unser Verlangen nach Glück kommt und wie man glücklich wird“. - ,Vielleicht wißt ihr zu wenig?“ - „Nein“, sagt sie, „Wir wissen, warum der Mensch Mythen und Religionen psychologisch braucht. Ich weiß, wie mein Genom sequenziert ist und was es aus mir macht. Doch warum bin ich so selten heiter?"

„Ich sehe“, sagt Demeter, ,euch mangelt es an Eudämonologie. Ihr habt die Kunst des Glücklichseins nicht erlernet. Im Wissen allein liegt noch nicht das Glück, eher in der Freiheit, die Wissen schenkt. Ihr müßt noch etwas hinzutun. Trillert etwa die Lerche ihr Lied, wenn sie im Grase bleibt? Tanzt etwa das Herz, wenn es in Bibliotheken brütet, vor Computern hockt? Euer Lebensalltag ist durch Wissenschaft und Technik so angstfrei und unbeschwert geworden. Da könnt ihr euch leicht aus eurem Lebensalltag befreien. Setzt den Abgründen ein Dennoch entgegen. Tut und denkt, was euch heiter macht. Lernt die Wege dazu. Steigt auf die Bergesgipfel. Singt euer Lied. Es muß nicht gleich das des Dionysos trunkenen Zarathustra am Steine in Sils-Maria sein. Singt das Lied der Heiterkeit des Geistes. Denn dies habt ihr uns Göttern voraus: Eure Seele kann heiter, ja glücklich sein, wenn ihr es nur recht erlernet. Und ihr habt nur dieses eine, euer Leben. - Gehet also und schaut, ob der Sizilianische Himmel nun für euch noch ein wenig heiterer sein kann.“

\section{Literatur}

[1] Assmann, Jan: Moses der Ägypter. Carl Hanser Verl. München 1998

[2] Börner, G. u.a (Hrsg.): Vom Urknall zum komplexen Universum. Serie Piper, Bd. 6, Veröffl. der Siemens-Stiftung, Piper, München 1993

[3] Breuer, H.: „Ich denke, also bin ich“, Leib und Seele, ein Jahrtausendkonflikt. Heitkamp Edition: Wir in unserer Welt 1998

[4] Damasio, A.: Ich fühle, also bin ich. List Verl. München 2000 
[5] Delsemme, Armand: Our cosmic origin. Cambridge Univ. Press. 1998

[6] Downs, R.: Books that changed the world. Mentor, USA 1983

[7] Eliade, Mircea: Kosmos und Geschichte. Insel Verlag Taschenb. Nr. 1580, 1994

[8] ERLER, M.: Leben wie ein Gott auf Erden. Epikur oder: Wie man glücklich wird. ZEIT 27, 1.7.1999

[9] Friedenthal, F.: Goethe. Piper Verl. München 1963

[10] Gierer, A.: Im Spiegel der Natur erkennen wir uns selbst. Rowohlt 1998

[11] HäGELE, P.: Was the Universe made for man? 1998

[12] Leaman, O.: Key Concepts in Eastern Philosophy. Routledge, London 1999

[13] Marcuse, Ludwig: Philosophie des Glücks. Diogenes Verl. Zürich 1972

[14] MarkL, H.: The future of nature. Europ. Review, Acad. Europaea Vol. 7 1999, 359 - 369.

[15] MarkL, H.: Dolly und die Folgen: Ist der Mensch wirklich ein Schaf? Vortrag Übersee Club Hamburg 2000

[16] Meier, Heinrich (Hrsg.): Die Herausforderung der Evolutionsbiologie. Serie Piper, Bd. 1, Veröffl. der Siemens-Stiftung, Piper, München 1988

[17] Meier, Heinrich u. Ploog, D.: Der Mensch und sein Gehirn, die Folgen der Evolution. Serie Piper, Bd. 7, Veröffl. der Siemens-Stiftung, Piper, München 1997

[18] Mittelstraß, J. (Hrsg.): Enzyklopädie Philosophie und Wissenschaftstheorie, J.B. Metzner Verl. Bd. 1 - 4, 1995/96

[19] Popper, K.: Alles Leben ist Problemlösen, Piper, München 1996

[20] Rотн, G.: Geist ohne Gehirn? Forschung und Lehre 5/2000

[21] Schmid, Wilhelm: Heiterkeit, ZEIT 41, 7.10.1999

[22] Schopenhauer, A.: Die Kunst, glücklich zu sein. Verl. C.H. Beck, München 1999

[23] SENECA: Vom Glückseligen Leben. (Heinrich Schmidt Hrsg.) Alfred Körner Verl. Stuttgart 1978

[24] Weniger, G.-Chr.: Projekt Menschwerdung. Heitkamp Edition: Wir in unserer Welt. 2000

[25] Wer deutet die Welt. Streitgespräch: Wolf Singer u. Lutz Wingen. ZEIT Nr. 50, 7.12.2000

Diverse Aufsätze in: TIME-Magazin, National Geographic

Nachträglich zur Kenntnis gelangt:

[26] Vollmer, G.: Die vierte bis siebte Kränkung des Menschen - Gehirn, Evolution und Meschenbild. In: Auf der Suche nach der Ordnung. Beiträge zu einem naturrealistischen Welt- und Menschenbild. Hirzel, Wiss. Verlagsges. Stuttgart 1995, S. 43 - 59 